MITSUBISHI ELECTRIC RESEARCH LABORATORIES

http://www.merl.com

\title{
The ŹircusĆoncept Sketch for a Learning Environment and Online Community
}

\author{
Carol Strohecker
}

TR94-22 December 1994

\begin{abstract}
This paper describes premises of a video about an as-yet unimplemented design for a virtual environment. The video is a basis for discussion - a sketch, rather than a specification, of a virtual playspace and interactions that can happen there. The playspace is conceived as a l̈earning environmentïn which activities can focus thinking on certain sets of ideas. Conversations within the multiuser environment can also be conducive to learning, and to developing an önline community.T̈hese actions and conversations would depend on a future system combining technologies in networked graphics, speech, AI, various input devices, and high-level software for constructing animations and audio/video sequences.
\end{abstract}

Presence: Teleoperators and Virtual Environments, Vol. 6, No. 3, 1997, pp. 339-349

\footnotetext{
This work may not be copied or reproduced in whole or in part for any commercial purpose. Permission to copy in whole or in part without payment of fee is granted for nonprofit educational and research purposes provided that all such whole or partial copies include the following: a notice that such copying is by permission of Mitsubishi Electric Research Laboratories, Inc.; an acknowledgment of the authors and individual contributions to the work; and all applicable portions of the copyright notice. Copying, reproduction, or republishing for any other purpose shall require a license with payment of fee to Mitsubishi Electric Research Laboratories, Inc. All rights reserved.
} 



\section{$1 \quad$ Designing Starting Points}

Interest in combining certain technologies as a platform for learning environments has influenced the formulation of a now-imaginary virtual space called the "Zircus." Characterized through a video of the same name, this space is part zoo and part circus. Animals are not trained to perform unnatural tricks, but roam freely through familiar terrains, in the spirit of the well designed zoos. Visitors are not mere spectators, but creators of acrobatic routines, juggling sequences, and other circus arts. Friends from all over the globe can see themselves represented in cartoon-like form - often, forms that they themselves design. As they wander through various landscapes and interiors, these new-age "pen pals" can converse in different languages with each other and with resident characters ("agents"). The visitors can also compose and send messages to one another, often drawing from a multimedia database, and invent their own creatures to dwell in the Zircus. Most of the visitors are probably young people, but that is not a rule.

This tall order for an implementation stems from discussions at Mitsubishi Electric Research Laboratories (MERL) in which researchers strove to work toward two goals. One was a broad aim, to develop "environments for learning and play." Because of the research interests of the group, we imagined these environments as bringing together certain key technologies: real-time, networked 3D graphics; speech recognition and generation; natural language processing; and artificial agents (Rich et al., 1994). The Zircus is a suggestion of how these research domains could come together as supports for an environment that fosters human learning, as well as fundamental research in learning.

In its dynamic form, the video sketch goes beyond the researcher's paper or the architect's drawing. The video enables portrayal not only of landscape, structures, and interiors, but also of characters and types of interactions. As architects would use sketches to facilitate discussions among colleagues and clients, researchers at MERL are using the Zircus video to further ideas about technological supports for learning environments and online communities. The video is a starting point for further sketches and experimental prototypes, but even a fully implemented environment would be just a starting point. Ultimately, visitors to the virtual environment become its architects.

\section{$2 \quad$ A "Constructionist" Virtual Environment}

In "The Lessons of Lucasfilm's Habitat," Morningstar and Farmer (1991) describe their experiences in designing and implementing a base for a graphical online community. Some of their discoveries are strikingly consonant with principles of good learning environment design. One point is their shift of focus from the technology that supports a system to the participants who develop an online community through their use of the system:

The essential lesson that we have abstracted from our experiences with Habitat is that a cyberspace is defined more by the interactions among the characters within it than by the technology with which it is implemented. While we find much of the work presently being done on elaborate interface technologies - DataGloves, head-mounted displays, special-purpose rendering engines, and so on - both exciting and promising, the almost mystical euphoria that currently seems to surround all this hardware is, in our opinion, both excessive and somewhat misplaced. We can't help having a nagging sense that it's all a bit of a distraction from the really pressing issues. At the core of our vision is the idea that cyberspace is necessarily a many-participant environment. It seems to us that the things that are important to the inhabitants of such an environment are the capabilities available to them, the characteristics of the other people they encounter there, and the ways these various participants can affect one another.

(Morningstar and Farmer, 1991, p.274) 
They conclude that "one of the goals of a next generation Habitat-like system ought to be to permit far greater creative involvement by the participants without requiring them to ascend to full-fledged guruhood to do so" (Ibid., p.295).

This emphasis on interactions, both social and instrumental, that can happen in an environment, is consistent with my use of the term "learning environment." This view begins with Papert's (1980) discussion of "microworlds." Such domains consist of elements that embody fundamental properties of some conceptual area. By working with the elements, people get in touch with core ideas and develop understandings of the larger topic. For example, in the microworld of "turtle geometry" (see Abelson and diSessa, 1980; Papert 1980), children draw pictures by directing the movements of a graphical object that looks like a turtle. The turtle has two properties: a position and a heading. By creating pictures through manipulations of these two basic properties, children can come to understand the concept of vector. This building-block idea can, in turn, help in developing further understandings - of angles, the geometries of squares and spirals, etc.

The act of working - or playing - with the ideas is crucial. It is through the act of making something, of constructing something personally meaningful, that connections between new ideas and existing understandings can happen. In this model of learning, knowledge is something that grows from what a person already understands. It isn't something that can just be "poured" into the head or "transferred" from one person to another. Papert has elaborated on this principle in his discussion of "constructionism" (Harel and Papert, 1991; Papert, 1990, 1993). The word is a play on Piaget's term "constructivism," adopted

to capture the sense in which the child must make and remake the basic concepts and logical thought-forms that constitute his intelligence. Piaget prefers to say that the child is inventing rather than discovering his ideas. ... The ideas in question do not preexist out there in the world, only awaiting their discovery by the child: each child must reinvent them for himself. By the same token, since the ideas have no a priori external existence, they cannot be discovered by simple exposure; rather, they must be constructed or invented by the child. (Gruber and Vonèche, 1977, p. xxxvii)

Papert's extension of this view is that the child's inventing of the ideas can be facilitated by actually constructing an object in the world. The learner may be a child but may also be an older newcomer to an idea.

A learning environment is a context in which constructive activities can happen. The context is conceptual, technical, and social. People work with specifically designed or selected tools or media. Often, they work together; often, for extended periods of time. They develop not only individual understandings, but a culture that supports the learning.

Learning researchers are interested in the processes by which people come to understand given phenomena. In order to glean data on these processes, the researchers design tools and contexts for using them that can focus the thinking and activity on key ideas. Often, there is an emphasis on the learners' reflection, articulation, and modification of their own processes of learning (see Ackermann, 1988; Schön, 1983; Strohecker, 1991). In this sense, the learner also becomes a learning researcher, and there is an important connection between a learning environment and an environment for research on learning. The two might very well be the same. The designer of the learning environment can develop some initial conditions, but it is the learners themselves who do the work, shaping the environment along the way.

These ideas - that learners construct their own understandings and that environment designers can only establish conditions and set directions - are consistent with another of Morningstar and Farmer's lessons:

There were two sorts of implementation challenges that Habitat posed. The first was the challenge of creating a working piece of technology... The second challenge was the creation and management of the Habitat world 
itself. It is the experiences from the latter exercise that we think will be the most relevant to future cyberspace designers.

Initially, we were our own worst enemies in this undertaking, victims of a way of thinking to which all engineers are dangerously susceptible. This way of thinking is characterized by the conceit that all things may be planned in advance and then directly implemented according to the plan's detailed specification. For persons schooled in the design and construction of systems based on simple, well-defined, and well-understood foundation principles, this is a natural attitude to have. Moreover, it is entirely appropriate when undertaking most engineering projects. It is a frame of mind that is an essential part of a good engineer's conceptual tool kit. Alas, in keeping with Maslow's assertion that "to the person who has only a hammer, all the world looks like a nail," it is a frame of mind that is easy to carry beyond its range of applicability. This happens when a system exceeds the threshold of complexity above which the human mind loses its ability to maintain a complete and coherent model. (Morningstar and Farmer, 1991, pp. 284-85)

Learning environments designed for "educational" purposes can suffer from the same syndrome. The designer (or teacher or curriculum developer) who strives to maintain the role of "omniscient central planner who dictates all that happens" (Ibid., p. 299) is apt to find that real learning isn't one of them.

Morningstar and Farmer say that "...the line of development most interesting to us is to expand on the idea of making the development and expansion of the world itself part of the users' sphere of control" (Ibid., p.297). This goal and the framework of learning research guided my sketch of the Zircus as a learning environment. In an effort to join these criteria with the concerns of other researchers on the project, I drew from the architectural notion of "placemaking" (see, for example, Frampton, 1992) and the idea of "experience design" as Scott Fisher has defined it:

...it is not the hardware that people might use that will determine whether telepresence becomes a powerful and popular medium - instead, it will be the experiences that they are able to have that will drive its acceptance and growth. The central challenge for telepresence remains: What do you do when you get there? What actually occurs in a virtual world? What kinds of actions can a user take there? How does the world respond to what someone does in it? What kinds of things might happen? and What makes and keeps a virtual world interesting? These are issues of experience design.

Exploring the boundaries of these issues will launch this new medium far beyond its origins in photo-realistic computer graphics and traditional simulation. (Fisher, 1993, p.212)

The results of these combined concerns follow. Visitors to the Zircus find themselves in a world where it makes sense to question how people and animals move. Given the international populace, it also makes sense to become curious about unfamiliar languages. The sketch for the learning environment suggests activities that can support explorations of these areas. Of course, how users of a built system ultimately would incorporate such activities into their experiences is something that we, as direction-setters, can only imagine.

\section{Features of the Environment, Its Inhabitants, and Their Interactions}

The Zircus anticipates a platform with audio, video, and graphic output, but the modes of input are not so clear. Speech should be one of them, as should some sort of gestural input. The video assumes these facilities without showing how actions outside of the environment map to those inside of it. The emphasis is on what happens in the environment. As we watch the video, we see and hear things as though we were users, 
members of the online community. We follow the experiences of Jake, who enters the Zircus with his sister and then joins a friend in searching for an elusive talking horse.

\subsection{Who Am I?}

Upon entering the environment, users choose what garb (and presumably, what related persona) to don. The visitors we see choose clown costumes (see Figure 1). Later, they meet an acrobat and a stilt walker. Of course, the Zircus should have an array of choices in the forms of circus and animal characters. Some users, once inside the environment, might want to create their own costumes and characters for later visits. This activity is one of many possible ways in which users could extend the environment, a key consideration in providing for multiuser spaces (see Curtis, 1992, and Morningstar and Farmer, 1991).

The activity echoes a phenomenon that currently happens in so-called MUDs and MOOs: 1 in these text-based multiuser environments, many participants adopt pseudonyms that indicate something about who they are or how they would like to be perceived. Some users actively experiment with alternate identities (see Curtis, 1992, and Rheingold, 1993).

From a psychological standpoint, this sort of experimentation could be a valuable feature of a learning environment (see Turkle, 1984, and Winnicott, 1971). Yet, in a system supporting an environment like the Zircus, certain technical feasibilities could interfere with the process rather than provide for it. It would be possible to texture-map users' likenesses on cartoon characters' faces, for example, so that participants could easily recognize each others' real-world selves. One of the compelling reasons for spending time in the environment could get lost, though. Many users are drawn to the opportunity of experimenting with a semi-anonymous situation in which they can actively construct a new identity.

\subsection{Where Am I?}

The Zircus "place" is a rolling landscape through which visitors represented as cartoon characters can wander around with robot and animal "residents" (see Figure 2). Characters meet, converse, and play in the hills and valleys of the landscape, as well as in and around structures that are on it: a stable, a circus tent, and a circle of trailers including a canteen, a mail stop, and a WC. ${ }^{2}$ In an implemented version of the Zircus, visitors would no doubt add additional areas and structures.

Characters get from one part of the Zircus to another by walking - indeed, human and animal locomotion are a focus of the learning environment. A helpful accessory would be a dynamic "you are here" sort of map, which tracks characters as they move about and shows their location on a schematic view of the Zircus layout. Riding an animal like a horse can afford an opportunity to change point of view or experiment with different gaits and speeds of movement through the environment.

Every now and then, adjustments of lighting and sound could create the effects of rain and other weather changes.

\subsection{Who Else Is Here?}

Visitors to the Zircus encounter other live participants represented as animals or circus characters. They also meet permanent residents of the environment, free-roaming agents that have particular personalities and capabilities (see Figure 3). Agents sometimes speak with each other and with visitors to the environment.

\footnotetext{
${ }^{1}$ MUD stands for "MultiUser Dungeon"; MOO means "MUD Object-Oriented." These loosely structured games typically have some associated programming language with which participants can add areas and objects to the environment.

${ }^{2}$ Certain uses of the virtual canteen and WC are the subject of future research.
}

5

Community 
Many of the discussions that led to the Zircus video reflected MERL researchers' interests in an environment that could support learning a foreign language. Conceptions of such an environment ranged from next-generation audio courses with added features such as modeling users' skill levels and directing output accordingly, to language-lab style applications updated with interactive video and computational language parsing, to more game-like interpretations.

The Zircus sketch reflects a particular approach to the issue of foreign-language learning. It assumes that the Zircus environment is not the sole source of information about the language or practice with it. It also assumes that whatever foreign-language learning happens in the multilingual environment will occur through participants' desires to communicate with each other. In the course of conversations, they can try to make themselves understood and help one another discover expressions, vocabularies, etc.

The Zircus is not an attempt to duplicate aspects of culture, as some foreign-language instructors might advocate. Rather, the Zircus is a place where visitors form their own culture based on who they meet, what they find, and what they do. The fact of its multilingual populace is likely to encourage exchanges in a variety of languages. For these reasons, the environment should be regarded as a container of possibilities for supplemental, self-initiated practice rather than a medium for directed foreign-language learning. Much of the practice happens in the course of doing other things that a user considers important.

However, given the international and multicultural participation, a salient characteristic of the environment would be its variety of spoken languages. The sketch includes some builtin supports for visitors looking for ways to communicate with new friends. Each agentcharacter embodies some function that can help in practicing or understanding a foreign language.

Three of the characters are straightforward embodiments of one specific language. There is an English-speaking horse, ${ }^{3}$ a Spanish-speaking robot, and a French-speaking robot. These characters would be endowed with some limited set of words and conditions for their use. The video also portrays a multilingual robot who is a savvy guide, familiar with the environment and its inhabitants. He is something of an idiom machine as well. In response to direct questions or triggered by keywords in conversation, he explains idiomatic expressions and shows illustrations of them in his TV-like eyes. This robot has a talkative parrot friend (see Figure 4). Their relationship is reminiscent of symbiosis; rarely are they seen apart. The parrot is basically a repetition machine, a way to give Zircus visitors a second hearing of spoken words and phrases.

Eventually the environment might include a number of characters embodying vocabularies in different languages and devices to assist in language-learning. We might imagine a rhyming machine, for example (a mockingbird?), a (bad) pun machine (a hyena?), a songbird, or even a robot that doesn't hear well, so that users find themselves saying things to it again or in different ways. There might be a robot that mumbles multilingual conversation fillers like "umm" and "let's see..."

Such characters would function as a kind of multilingual "spice." The lighthearted characterizations could be a forgiving cover to technological limitations in natural language processing.

\subsection{What Can We Do Here?}

With high-level software accessible from different areas of the Zircus, users can work with certain ideas and construct objects, characters, and other additions to the environment (see Figure 5). Extending the environment is one way in which users can "appropriate" it, or make it feel like it is theirs, in some sense. Developing a sense of ownership or attachment to an environment can be an important condition for learning about the ideas it embodies (see Papert, 1980, 1993).

\footnotetext{
${ }^{3}$ Not Ed! His name is Ned.
}

6

Community 
A creature construction set becomes a microworld for working with fundamentals of motion, such as balance and center of mass, as they relate to how animals move (see Figure 6). Other activities involve explorations of motion as it relates to circus arts (see Figure 7). A simulated stilt walker, for example, can become another microworld for balance. By changing lengths of the stilts and seeing the immediate effects of each change, users can get a sense for how this parameter affects the overall balance of a system. By controlling when a ball is tossed, how quickly it moves, how high it goes, or other parameters in a juggling simulation, users can get a "big picture" view of the coordinated event that would otherwise be difficult to manage in learning to juggle. Similarly, choreographing an acrobatic routine can be a way of learning relationships between strength, speed, and other characteristics that determine a sequence of stunts.

Physical realism is not the goal of such simulations. For purposes of a learning environment, the "reality" can be pared down or even recast to enable focus on a few fundamental properties. The selection of such properties, as well as the consistency and coherence of their operation, are more important than duplicating real-world conditions.

Furthermore, the simulations are not meant to substitute for developing physical skills and the kinds of understandings they engender. There is a sense in which three balls, a big room, and a good coach, for example, will always be the best learning environment for juggling. But a computer simulation can supplement and build from intuitions that are rooted in this kind of real-world experience. The computer can enable repetitions, different views, and other variations in the experience of a phenomenon, lending a richness that helps in constructing an understanding of it. As the saying goes, we don't really understand something until we understand it in a few different ways.

Visitors to the Zircus can interact with other members of the online community even when they are not present simultaneously. Participants can send each other messages, which can be retrieved at the mail stop (see Figure 8) or delivered by a messenger-pigeon agent that shows the messages in its TV-like eyes. Another of the constructive activities in the environment is composing such messages, which may consist of both sound and pictures (see Figures 9 and 10). The implication is that users can draw from databases of still images, moving images, sounds, and music in composing messages. In this sense, the environment is also something of a reference tool, bearing similarities to the "knowledge machine" that Papert (1993) describes. Ideally, users would also be able to import their own home video as well, which would provide another opportunity for them to "appropriate" the environment. In any case, they would need software tools for searching and otherwise navigating through these media pieces, as well as for manipulating and assembling them into messages.

\section{Further Work}

Obviously, we are a long way from realizing a comprehensive environment such as this. There are many obvious needs, most of which the entire field is facing. Envisioning a context like the Zircus, though, helps in identifying starting points and areas of emphasis.

The concepts suggested in Zircus lead naturally to further sketches and a number of experiments. Some of these will be in the realm of online communities. Others will involve software microworlds that can be embedded at different sites of a Zircus-like environment. Still others may involve techniques such as fast texture-mapping and incorporation of geographic survey data. The road from sketch to implementation will include iterative changes based on technical feasibility and work with people using prototypical systems and software.

In the midst of all of these efforts, we have to remember that a working system eventually will support a community whose character and pastimes will be defined more by it members than by any plan we can devise. 


\section{Figures and Captions}

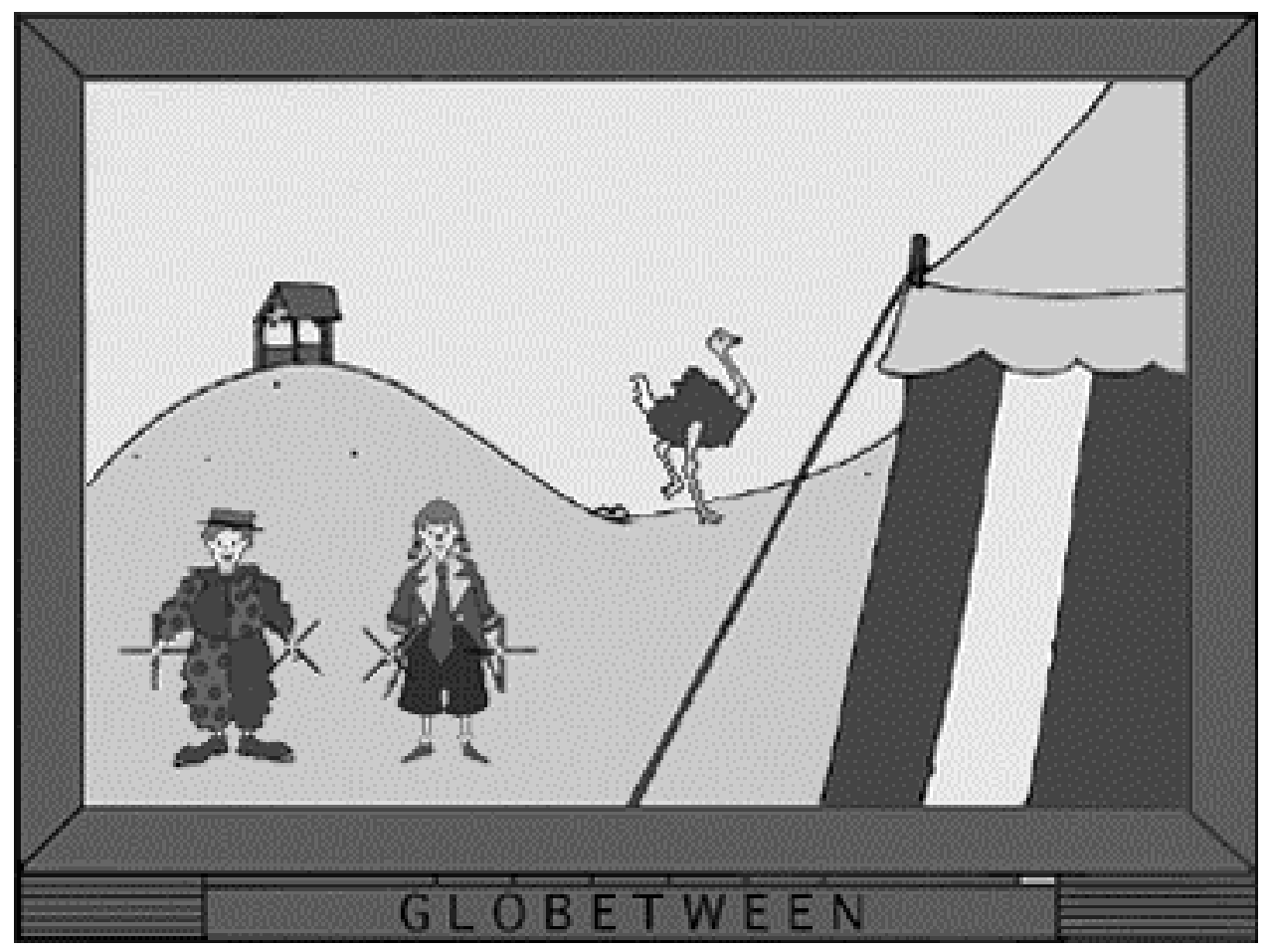

Figure 1. The "Zircus" is a thought experiment in virtual world design, not yet a built environment. We sketched it as a playspace in which users from all over the globe can converse, explore varied terrains, and construct characters, circus-arts routines, and multimedia messages.

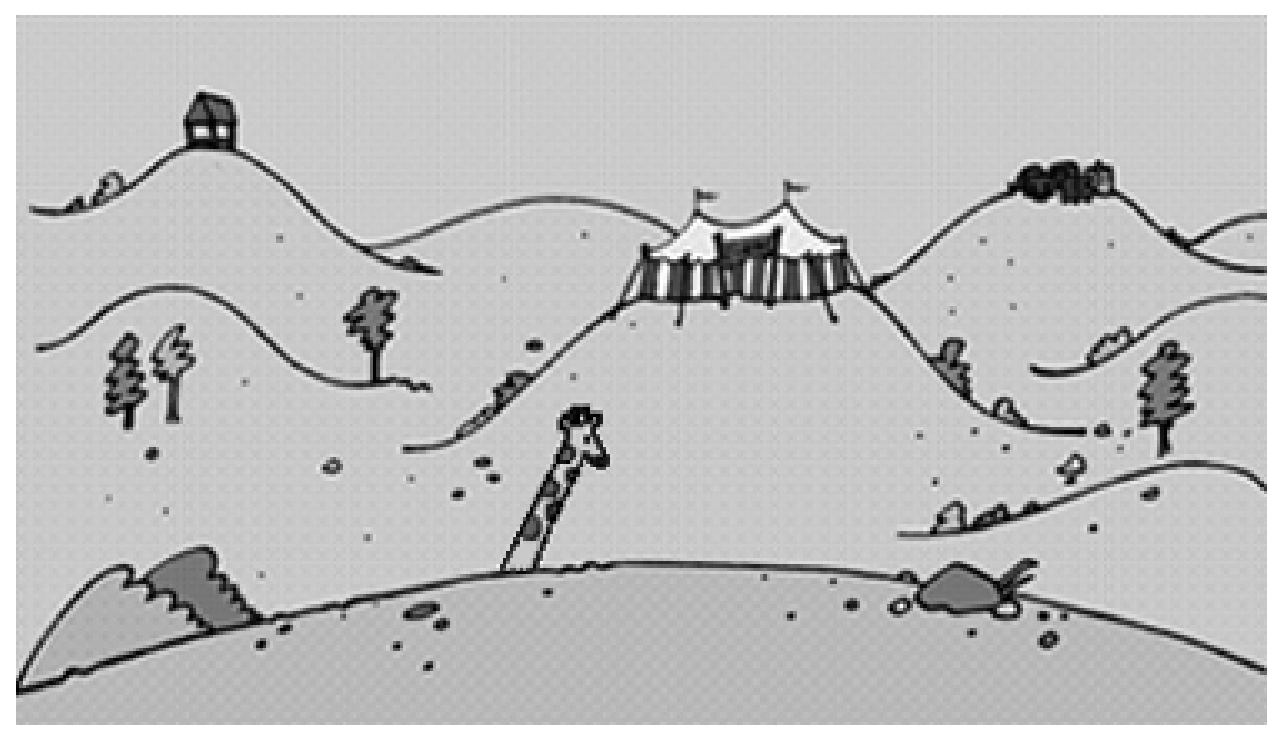

Figure 2. The landscape mimics that of a well designed zoo - animals can roam freely through terrain like that of their natural environs, sculpted to contain them without cages. 

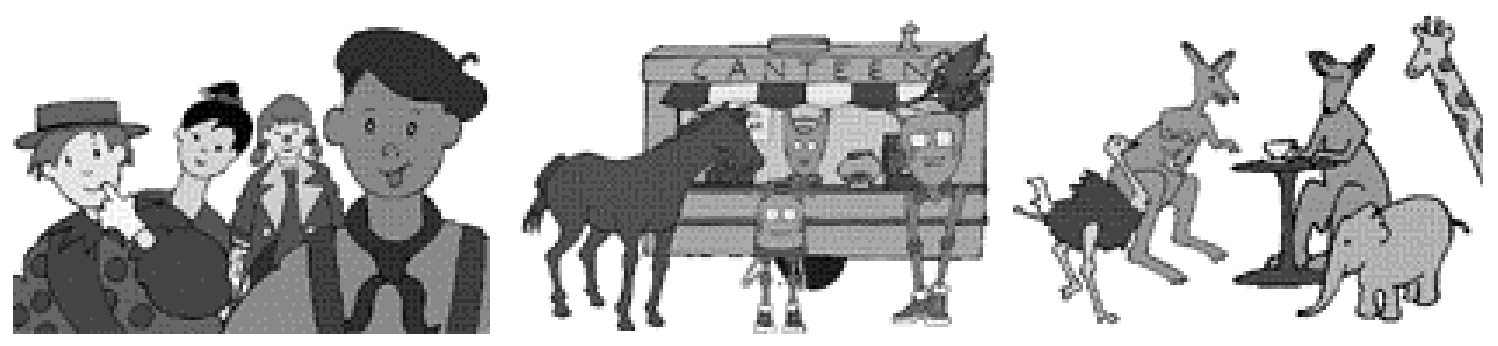

Figure 3. Members of the online community include visitors as well as permanent residents - humans, robots, and animals. The visitors can talk with each other and, using a more limited vocabulary, with some of the residents. Ideally, users could construct representations of themselves and other characters.

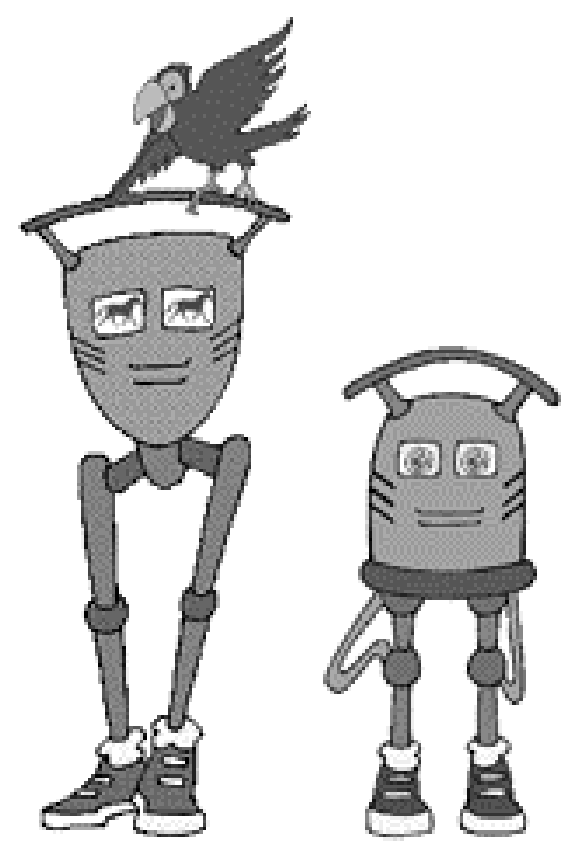

Figure 4. All manner of odd creatures live in the Zircus. Some of them are "agents" programmed to perform specific functions, such as the parrot that is simply a "repetition machine." By repeating phrases at certain times, it gives users another hearing of unfamiliar expressions or pronunciations. Robot characters have similar specialties and sometimes show video or other images in their TV-like eyes. 

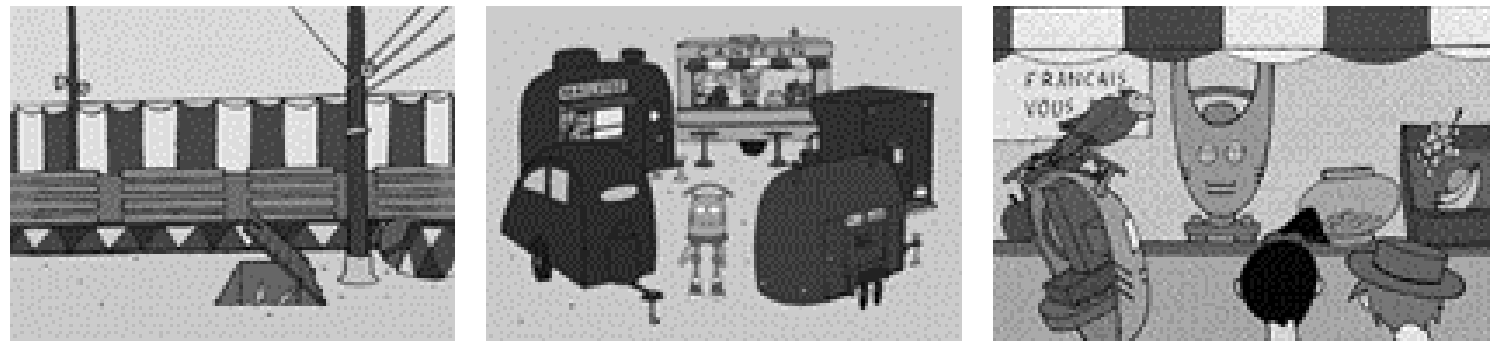

Figure 5. As visitors wander through different parts of the Zircus, they find different things to do and characters to interact with. The interactions are not structured like games, but depend on individuals' curiosities about people and objects.

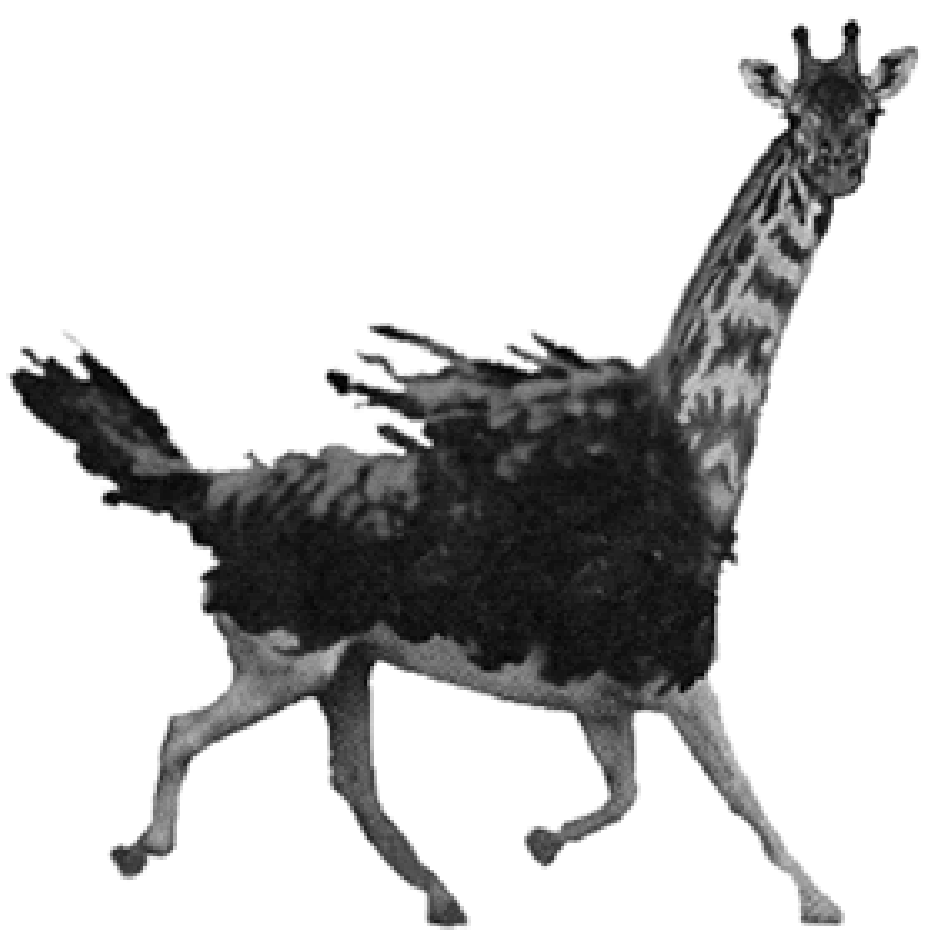

Figure 6. Among the high-level software is a "construction kit" enabling users to create whimsical creatures and experiment with their modes of locomotion.

Horse legs, ostrich body: Eadweard Muybridge, (01957 Dover Publications, Inc.

Giraffe neck: Helen \& Frank Schreider, (C) National Geographic Society 


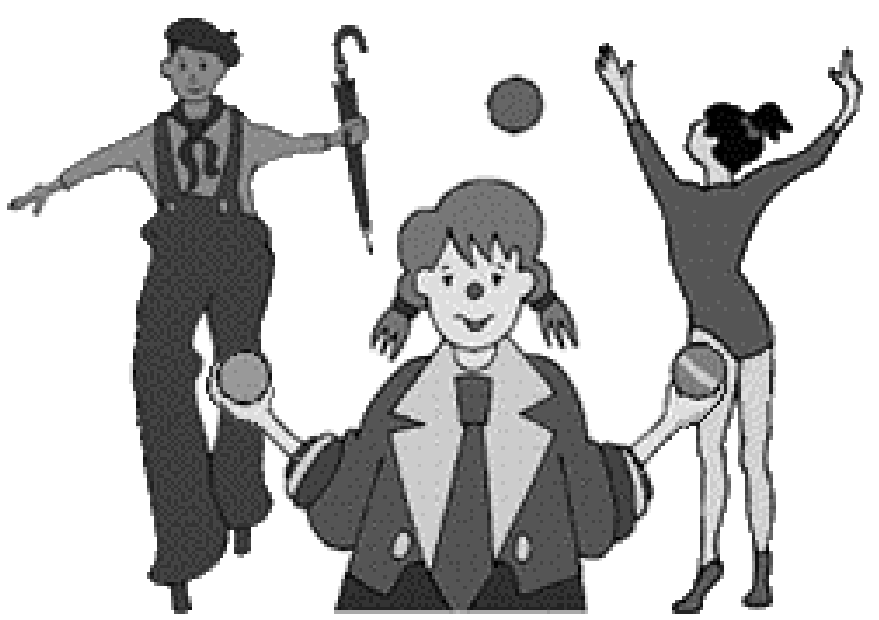

Figure 7. By constructing motion sequences, users can supplement real-world experiments with circus arts such as stilt walking, juggling, and acrobatics. (How does the balancing change if the stilts get longer? What are some variations on toss patterns? How does speed affect the height of a jump?)

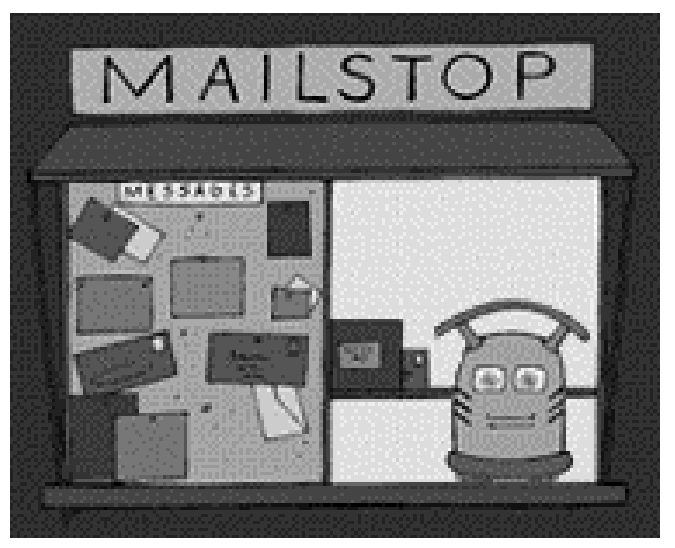

Figure 8. Friends will not always find each other in the environment, but they can leave aural and visual messages at the mailstop. 


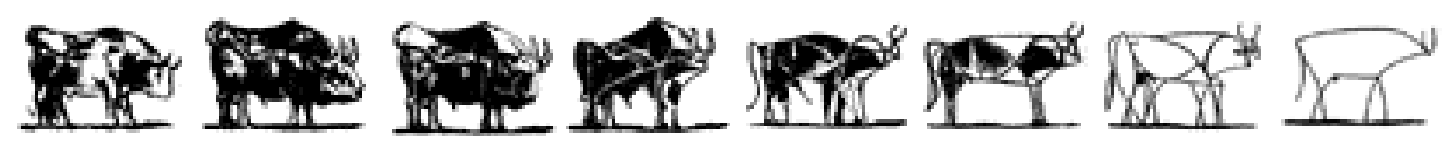

Figure 9. One of the messages shown in the video is a morph of this series by Picasso.

Picasso Bull states 1, 3-7, 9, 11; (C1993 ARS, NY/SPADEM, Paris

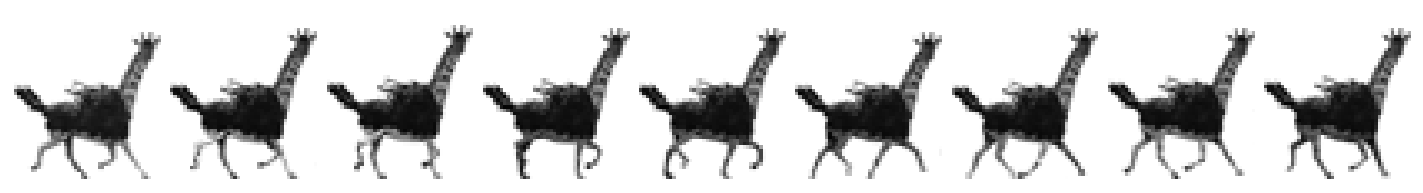

Figure 10. Another message shows the outcome of an experiment with a composite creature that has the legs of a horse, the body of an ostrich, and the head of a giraffe. The (purportedly) too-long neck throws the creature off-balance. Software to create experiments with balancing creatures is one of the follow-on projects related to Zircus.

Horse legs, ostrich body: Eadweard Muybridge, (C1957 Dover Publications, Inc. Giraffe neck: Helen \& Frank Schreider, (C) National Geographic Society

\section{Acknowledgments}

The Zircus video alludes to several research agendas, which came up in discussions with colleagues at Mitsubishi Electric Research Laboratories: Andrew Golding, Marc H. Raibert, Charles Rich, Yves Schabes, and Richard C. Waters. William T. Freeman, Michal Roth, and Mark Torrance later joined the group; Joseph Bates and Janet Murray also contributed to these discussions from time to time. Seymour Papert and Marc Raibert provided additional helpful conversations. I would like to thank each of these people for their contributions, many of which were inadvertent. Thanks also to Edith Ackermann, Kathleen Biddick, Larry Friedlander, Erin Hoffer, Ken Kaplan, and Sherry Turkle, who kindly provided comments after completion of the production.

Many people were involved in producing the video, and I would like to thank all of them again: Steve McNeon co-designed the visuals with me and coordinated the animation with 
John Shiple, who developed most of the sequences in MacroMind Director. Other contributions to production came from Peter Beebee, David Brann, Aaron Falbel, Colin B. McKee, Brian Robertson, Ilene Sterns, and Elizabeth Victor. Bob Aiudi, David Berlekamp, Mario Bourgoin, Leah Dwyer, Aaron Falbel, Andrew Golding, Tim Keefe, Brandon MacDowell, Hide Ohkami, Noriko Ohkami, Becca Rich, and Richard C. Waters lent their voices; Jenny Berlekamp, Gilda Keefe, Ceasar MacDowell, and Candy Sidner helped their children contribute to the soundtrack. Susannah Sheffer narrowly escaped enlistment. We did the recording and post-production with a cheerful and competent group at Multivision, Inc., of Needham, Massachusetts.

This work and related ongoing efforts are supported by Mitsubishi Electric Research Laboratories, Inc.

\section{References}

Abeill'e, A., \& Schabes, Y. (1989.) Parsing idioms in tree adjoining grammars. In Fourth Conference of the European Chapter of the Association for Computational Linguistics (EACL'89), Manchester.

Abeill'e, A., \& Schabes, Y. (1990.) Non compositional discontinuous constituents in tree adjoining grammar. In Proceedings of the Symposium on Discontinuous Constituents, Tilburg, Holland.

Abeill'e, A., \& Schabes, Y. (1992.) Non compositional discontinuous constituents in tree adjoining grammar. In Discontinuous Constituency. Mouton.

Abelson, H., \& diSessa, A. (1980). Turtle Geometry: The Computer as a Medium for Exploring Mathematics. Cambridge: MIT Press.

Ackermann, E. K. (1987.) Pathways Into a Child's Mind: Helping Children Become Epistemologists. Paper presented at the symposium, "Science Learning in the Informal Setting," Chicago Academy of Sciences.

Austin, H. A. (1976). A Computational Theory of Physical Skill. Ph.D. diss., Massachusetts Institute of Technology.

Bates, J. (1992). Virtual Reality, Art, and Entertainment. Presence, vol. 1., no. 1, pp. 13338.

Beilin, H., \& Pufall, P. B., eds. (1992). Piaget's Theory: Prospects and Possibilities. Hillsdale: Lawrence Erlbaum Assoc.

Blum, G., \& Salas, N. (1989.) Les Idiomatics: Français-Anglais, English-French. Éditions du Seuil.

Curtis, P. (1992). Mudding: Social Phenomena in Text-Based Virtual Realities. Proceedings of the 1992 Conference on Directions and Implications of Advanced Computing, Berkeley. Also available as Xerox PARC technical report CSL-92-4.

Fisher, S. S. (1993). Menagerie. Computer Graphics Visual Proceedings. ACM SIGGRAPH.

Frampton, K. (1980, 1992). Modern Architecture: A Critical History. London: Thames \& Hudson.

Gruber, H. E., \& Vonèche, J. J., eds. (1977). The Essential Piaget. New York: Basic Books, Inc.

Harel, I., \& Papert, S., eds. (1991). Constructionism. Norwood: Ablex.

Jenkins, H. (1993). "x Logic": Repositioning Nintendo in Children's Lives. Quarterly Review of Film and Video, vol. 14, no. 4, pp. 55-70.

Morningstar, C., \& Farmer, F. R. (1991). The Lessons of Lucasfilms' Habitat. Cyberspace: First Steps. ed. Michael Benedikt. pp.273-301. Cambridge: MIT Press.

Oldenburg, R. (1989). The Great Good Place: Cafés, Coffee Shops, Community Centers, Beauty Parlors, General Stores, Bars, Hangouts and How They Get You through the Day. New York: Paragon House. 
Papert, S. (1980). Mindstorms: Children, Computers, and Powerful Ideas. New York: Basic Books, Inc.

Papert, S., \& Associates. (1987). Learning at the Zoo: A Design for an EcoLearning Center at the St. Louis Zoo. Cambridge, MA: MIT Epistemology \& Learning Group.

Papert, S. (1990). A Unified Computer Environment for Schools: A Cultural/Constructionist Approach. Proposal to the National Science Foundation. Cambridge, MA: MIT Epistemology \& Learning Group.

Papert, S. (1991). New Images of Programming: In Search of an Educationally Powerful Concept of Technological Fluency. Proposal to the National Science Foundation. Cambridge, MA: MIT Epistemology and Learning Group.

Papert, S. (1993). The Children's Machine: Rethinking School in the Age of the Computer. New York: Basic Books.

Rheingold, H. (1993). The Virtual Community: Homesteading on the Electronic Frontier. Reading: Addison-Wesley.

Rich, C., Waters, D., Schabes, Y., Strohecker, C., Freeman, B., and Roth, M. (1994). "Demonstration of an Interactive Environment for Collaboration and Learning," IEEE Computer, vol. 27, no. 12.

Rich, C., Waters, D., Schabes, Y., Freeman, B., and Roth, M. (1994). "An Animated Online Community with Artificial Agents and Spoken Interaction," IEEE Multimedia, vol. 1, no. 4.

Schön, D. (1983.) The Reflective Practitioner. New York: Basic Books.

Sorkin, M., ed. (1992). Variations on a Theme Park. New York: Hill \& Wang.

Strohecker, C. (1991). Why Knot? Ph.D. diss., Massachusetts Institute of Technology.

Strohecker, C. (1992). Ideas for a System Supporting Users' Learning of a Foreign Language. MERL Note 92-19. Cambridge: Mitsubishi Electric Research Labs.

Turkle, S. (1984). The Second Self: Computers and the Human Spirit. New York: Simon \& Schuster.

Winnicott, D. W. (1971). Playing and Reality. London: Tavistock Publications. 